\title{
Bi-Objective Bilevel Programming Problem: A Fuzzy Approach
}

\author{
S. HASEEN AND A. BARI
}

\begin{abstract}
:
In this paper, a likely situation of a set of decision maker's with bi-objectives in case of fuzzy multi-choice goal programming is considered. The problem is then carefully formulated as a bi-objective bilevel programming problem (BOBPP) with multiple fuzzy aspiration goals, fuzzy cost coefficients and fuzzy decision variables. Using Ranking method the fuzzy bi-objective bilevel programming problem (FBOBPP) is converted into a crisp model. The transformed problem is further solved by adopting a two level Stackelberg game theory and fuzzy decision model of Sakawa. A numerical with hypothetical values is also used to illustrate the problem.
\end{abstract}

Additional Key Words and Phrases: Bi-Objective Bilevel Programming Problem, Multichoice Goal Programming, Trapezoidal Fuzzy Numbers, Ranking Function, Fuzzy Decision Model

\section{INTRODUCTION}

Bilevel Programming Problem (BPP) was first introduced by Bialas et al. [1980], they called it a two level programming problem with two levels of decision makers (DM), viz., the leader or first level decision maker (FLDM) and the follower or second level decision maker (SLDM). They also developed the Kth algorithm to solve their problem.

A BPP can be viewed as a standard mathematical program whose constraint region has been modified to include an implicitly defined function. To see this, suppose that each of the First and Second level decision maker wishes to maximize his own objective function, $F$ and $f$ respectively. As in the conventional setting FLDM has control over the set $X \subset \mathbb{R}^{n}$ and SLDM has control over the set $Y \subset \mathbb{R}^{m}$ where $X \cap Y=\phi$. It shall be assumed that FLDM has the first choice and selects $x \in X$, followed by SLDM who selects $y \in Y$, and that each objective function jointly depends on both of these choices; that is, $F, f: \mathbb{R}^{n} \times \mathbb{R}^{m} \rightarrow \mathbb{R}$. A further assumption is that the choice made by the FLDM may affect the set of feasible strategies available to the SLDM.

Bard [1982] defined BPP and solved the problem using grid search algorithm. There are many researchers who used different methods to get a better pareto optimal 
solution for the hierarchical problem of BPP. Among them are U.P. Wen [1986], Mathieu et al. [1994], Lu et al. [2005],Shi et al. [2005], Liu [1998] etc. who solved the BPP using different approaches.

Zadeh introduced fuzzy sets as sets with boundaries that are not precise. A fuzzy set can be defined mathematically by assigning to each possible individual in the universe of discourse a value representing its grade of membership in the fuzzy set. The fuzzy programming approach [Inuiguchi and Ramık [2000],Rommelfanger [1996], Slowinski and Teghem [1990]] is useful and efficient for treating a programming problem under uncertainty. While a classical and stochastic programming approach may cost a lot to obtain the exact coefficient value or distribution, fuzzy programming approach does not [Rommelfanger [2004]]. From this fact, fuzzy programming approach can be very advantageous when the coefficients are not known exactly. Fuzzy programming offers a powerful means of handling optimization problems with fuzzy parameters. Fuzzy programming has been used in different ways in the past. The fuzzy programming method for multi-objective programming problems was proposed by Zimmermann [1978] and has been advanced by Sakawa and colleagues [Sakawa [1993b]]. An interactive fuzzy satisfying method for multi objective linear programming problems and the interactive fuzzy decision making for multi-objective nonlinear programming using augmented minimax problems have been also introduced Luhandjula [1987], Sakawa [1984], Seo and Sakawa [1988]. The fuzzy programming method in which fuzziness in the decision making process is represented by using the fuzzy concept has also been studied extensively and many results have been published [Rommelfanger [1996], Teghem Jr et al. [1986]]. This method can be applied to not only the linear multi-objective but also the nonlinear multi-objective programming. There are many more authors who work on fuzzy problems some of them are Ganesan and Veeramani [2006], Allahviranloo et al. [2008], Ezzati et al. [2013] who have studied fuzzy linear program with triangular and trapezoidal fuzzy numbers

A new area of research was originated by Healy [1964] known as a multiple choice programming problem. In these problems there is a requirement to choose, among several possible combinations as an alternative to optimize an objective function subject to a set of constraints. It is a mixed binary programming in which all binary variables constitute a number of mutually exclusive choices where only one variable is to be selected. Multi-choice linear programming problem has been discussed by several authors such as Rav, Hiller and Lieberman [1990] etc. Chang [2007] proposed multichoice goal programming problem (MCGPP) in which 
objectives of mathematical programming problem can have multiple choices for their goals. Again, Tabrizi et al. [2012] proposed fuzzy goal programming for solving the MCGPP with multiple fuzzy aspiration levels and developed fuzzy membership function to get a compromise solution and recently [Biswal and Acharya [2009], Biswal and Acharya [2011]] consider the problem where the right hand side parameter has multiple choices.

In this paper the author has considered a case of BPP with two objectives at each level with multiple fuzzy choices for goals for every objective. They have further considered the cost coefficients and decision variables to be symmetric trapezoidal numbers. The problem is formulated and then crisp values using ranking functions are used to solve the formulation adopting the two planner Stackelberg function [see Anandalingam [1988]], the well-known fuzzy decision model of Sakawa [1993a] for a tolerance membership function.

\section{PRELIMINARIES}

Some basic definitions which are frequently used in the theory of BPP and fuzzy set theory.

For $x \in X \subset \mathbb{R}^{n}, y \in \subset \mathbb{R}^{m}, F: X \prod Y \rightarrow \mathbb{R}^{1}$ and $f: X \prod Y \rightarrow \mathbb{R}^{1}$, the linear bilevel programming problem (BLPP) can be written as follows:

$$
\begin{gathered}
\min _{x \in X} F(x, y)=c_{1} x+d_{1} y \\
\text { subject to } A_{1}+B_{1} \leq b_{1} \\
\min _{y \in Y} f(x, y)=c_{2} x+d_{2} y \\
\text { subject to } A_{2} x+B_{2} y \leq b_{2},
\end{gathered}
$$

where $c_{1}, c_{2} \in \mathbb{R}^{n}, d_{1}, d_{2} \in \mathbb{R}^{m}, b_{1} \in \mathbb{R}^{p}, b_{2} \in R^{q}, A_{1} \in \mathbb{R}^{p \prod n}, B_{1} \in \mathbb{R}^{p \prod^{m}}, A_{2} \in$ $\mathbb{R}^{q \prod^{n}}, B_{2} \in \mathbb{R}^{q \prod^{m}}$.

Definition 1(a) Constraint region of the linear BLP problem:

$$
S=\left\{(x, y): x \in X, y \in Y, A_{1} x+B_{1} y \leq b_{1}, A_{2} x+B_{2} y \leq b_{2}\right\}
$$

The linear bilevel programming problem constraint region refers to all possible combinations of choices that the leader and the follower may make.

Definition 1(b) Projection of $S$ onto the leader's decision space: 


$$
S(X)=\left\{x \in X: \exists y \in Y, A_{1} x+B_{1} y \leq b_{1}, A_{2} x+B_{2} y \leq b_{2}\right\}
$$

Definition 1(c) Feasible set for follower after every fixed $x \in X$ :

$$
S(x)=\left\{y \in Y: B_{2} y \leq b_{2}-A_{2} x\right\}
$$

Definition 1(d) Follower's rational reaction set for $x \in S(X)$ :

$$
P(x)=\{y \in Y: y \in \arg \min [f(x, \hat{y}): \hat{y} \in S(x)]\},
$$

where $\arg \min [f(x, \hat{y}): \hat{y} \in S(x)]=\{y \in S(x): f(x, y) \leq f(x, \hat{y}, \hat{y} \in S(x)\}$.

Definition 1(e) Inducible region:

$$
I R=\{(x, y):(x, y) \in S, y \in P(x)\}
$$

$\mathrm{S}$ is assumed non-empty and compact and for all decisions taken by leader, the follower has some room to respond i.e. $P(x) \neq \phi . P(x)$ defines response while $I R$ represents the set over which leader may optimize. The rational reaction set $P(x)$ defines the response while the inducible region $I R$ represents the set over which the leader may optimize his objective.

Thus in terms of the above notations, the linear BLP problem can be written as

$$
\min _{x \in X}\{F(x, y):(x, y) \in I R\}
$$

Even with the stated assumptions, problem may not have a solution.

Fuzzy Set: Let $A$ be the universe whose generic element be denoted by $a$. A fuzzy set $\tilde{A}$ in $A$ is a function $\tilde{A}: A \rightarrow[0,1]$.

Fuzzy number: A fuzzy number $\tilde{A}$ is a fuzzy set of the real line $A$, whose membership function $\mu_{\tilde{A}}(a)$ must posses the following properties with 


$$
-\infty \leq a^{(1)} \leq a^{(2)} \leq a^{(3)} \leq \infty
$$

$$
\mu_{\tilde{A}}(a)= \begin{cases}0 & a \leq a^{(1)} \\ \mu_{\tilde{L}(a)} & a^{(1)}<a \leq a^{(2)} \\ \mu_{\tilde{U}(a)} & a^{(2)}<a \leq a^{(3)} \\ 0 & a>a^{(3)}\end{cases}
$$

where $\mu_{\tilde{L}}(a):\left[a^{(1)}, a^{(2)}\right] \rightarrow[0,1]$ is continuous and strictly increasing and $\mu_{\tilde{U}}(a):\left[a^{(2)}, a^{(1)}\right] \rightarrow[0,1]$ is continuous and strictly decreasing.

Membership function $\mu_{\tilde{A}}(a)$ : We frequently define function $\tilde{A}$ and say that the fuzzy set $A$ is characterized by its membership function $\mu_{A}: A \rightarrow[0,1]$

Symmetric Trapezoidal Fuzzy Number (STFN): A symmetric trapezoidal fuzzy number can be completely specified by the foursome $\tilde{A}=\left(a^{(1)}, a^{(2)}, \gamma, \gamma\right)$ on real numbers $\mathbb{R}$ if there exist real numbers $a^{(1)}, a^{(2)} ; a^{(1)} \leq a^{(2)}$ and $\gamma<0$ such that the membership function is

$$
\mu_{\tilde{A}(a)}= \begin{cases}\frac{a+\gamma-a^{(1)}}{\gamma} & a^{(1)}-\gamma<a \leq a^{(1)} \\ 1 & a^{(1)}<a \leq a^{(2)}, \\ \frac{-a+a^{(2)}+\gamma}{\gamma} & a^{(2)}<a \leq a^{(2)}+\gamma \\ 0 & \text { otherwise }\end{cases}
$$

The symmetric trapezoidal fuzzy number (STFN) is depicted in the figure below

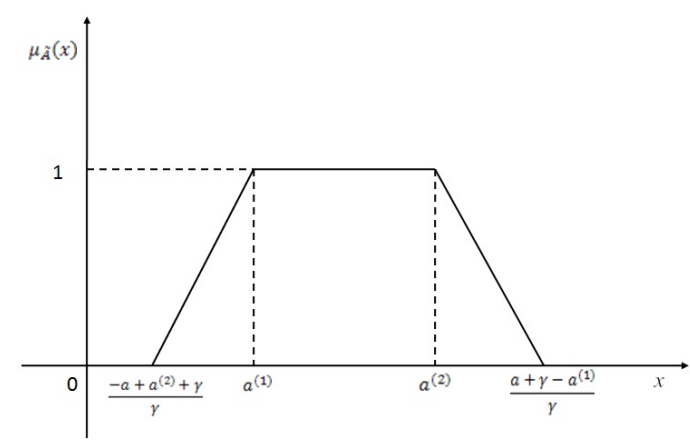

Ranking Function: If $\tilde{A}=\left(a^{(1)}, a^{(2)}, \gamma, \gamma\right)$ and $\tilde{A}=\left(b^{(1)}, b^{(2)}, \beta, \beta\right)$ are two STFN then their product can be defined as 
$\left(a^{(1)}, a^{(2)}, \gamma, \gamma\right) \times\left(b^{(1)}, b^{(2)}, \beta, \beta\right)$

$\left(\frac{a^{(1)}+a^{(2)}}{2}\right)\left(\frac{b^{(1)}+b^{(2)}}{2}\right)-\omega,\left(\frac{a^{(1)}+a^{(2)}}{2}\right)\left(\frac{b^{(1)}+b^{(2)}}{2}\right)+\omega,\left|a^{(2)} \beta+\gamma b^{(2)}\right|,\left|a^{(2)} \beta+\gamma b^{(2)}\right|$ where, $\omega=\frac{t_{1}-t_{2}}{2}$

$t_{1}=\min \left\{a^{(1)} b^{(1)}, a^{(1)}, b^{(2)}, a^{(2)} b^{(1)}, a^{(2)} b^{(2)}\right\}$

$t_{2}=\max \left\{a^{(1)} b^{(1)}, a^{(1)}, b^{(2)}, a^{(2)} b^{(1)}, a^{(2)} b^{(2)}\right\}$

\section{FORMULATION OF BI-OBJECTIVE BILEVEL PROGRAMMING PROBLEM (BOBPP)}

Again, the BPP can have more than one objective function at each level. So we consider here a linear bilevel programming problem with two objectives of maximization type at each level. Let the leader, FLDM have control over decision variable $x \in \mathbb{R}^{n}$ and the follower, SLDM, have $y \in \mathbb{R}^{m}$. Moreover, the decision variables are trapezoidal fuzzy number with fuzzy cost coefficients. Cost coefficients are also fuzzy trapezoidal number. Mathematically, Bilevel Programming Problem (BPP) with two objectives of maximization type and fuzzy parameters can be written as follows

For $\tilde{x} \in X \subset \mathbb{R}^{n}, \tilde{y} \in Y \subset \mathbb{R}^{m}$

$$
\begin{aligned}
& \left.\begin{array}{ll}
\max _{\tilde{x} \in X} \quad F_{1}(\tilde{x}, \tilde{y})=\tilde{c}_{1} \tilde{x}+\tilde{d}_{1} \tilde{y} \\
F_{2}(\tilde{x}, \tilde{y})=\tilde{c}_{2} \tilde{x}+\tilde{d}_{2} \tilde{y}
\end{array}\right\} \quad \text { where } \tilde{y} \text { solves } \\
& \text { subject to } \quad A_{1} \tilde{x}+B_{1} \tilde{y} \leq b_{1} \\
& \max _{\tilde{y} \in Y} \quad f_{1}(\tilde{x}, \tilde{y})=\tilde{c}_{3} \tilde{x}+\tilde{d}_{3} \tilde{y} \\
& f_{2}(\tilde{x}, \tilde{y})=\tilde{c}_{4} \tilde{x}+\tilde{d}_{4} \tilde{y} \\
& \text { subject to } \quad A_{2} \tilde{x}+B_{2} \tilde{y} \leq b_{2} \\
& \tilde{x} \geq 0, \tilde{y} \geq 0 \text {. }
\end{aligned}
$$

Now, in real life problem there may be situation where there are more than one choices in goals in such case the DM's of BOBPP have multiple fuzzy aspiration goals. Then BOBPP with multiple fuzzy aspiration goals may be formulated as

$$
\left.\begin{array}{ll}
\max _{\tilde{x} \in X} & F_{1}(\tilde{x}, \tilde{y})=\widetilde{G}_{1} \\
F_{2}(\tilde{x}, \tilde{y}) & =\widetilde{G}_{2}
\end{array}\right\} \quad \text { where } \tilde{y} \text { solves }
$$




$$
\begin{array}{ll}
\text { subject to } & A_{1} \tilde{x}+B_{1} \tilde{y} \leq b_{1} \\
\max _{\tilde{y} \in Y} & f_{1}(\tilde{x}, \tilde{y})=\tilde{g}_{1} \\
& f_{2}(\tilde{x}, \tilde{y})=\tilde{g}_{2} \\
\text { subject to } & A_{2} \tilde{x}+B_{2} \tilde{y} \leq b_{2} \\
& \tilde{x} \geq 0, \tilde{y} \geq 0 .
\end{array}
$$

where $\widetilde{G}_{i}, i=1,2$. are fuzzy aspiration gaols of FLDM and $g_{i}, i=1,2$. are fuzzy aspiration gaols of SLDM. Since there are fuzzy aspiration goals at each objective the fuzzy bi-objective bilevel goal programming problem (FBOBPP) can be written as different cases.

Case 1. When there are two fuzzy aspiration goals to choose the FBOBPP will be

$$
\begin{aligned}
& \max \quad \sum_{i=1}^{2} \mu_{F_{i}} \\
& \text { subject to } \quad \mu_{F_{i}} \leq 1-\left[\frac{F_{i}(\tilde{x}, \tilde{y})-G_{i}^{(1)}}{d_{1}^{-}} z_{1}+\frac{F_{i}(\tilde{x}, \tilde{y})-G_{i}^{(2)}}{d_{2}^{-}}\left(1-z_{1}\right)\right] \\
& \mu_{F_{i}} \leq 1-\left[\frac{G_{i}^{(1)}-F_{i}(\tilde{x}, \tilde{y})}{d_{1}^{+}} z_{1}+\frac{G_{i}^{(2)}-F_{i}(\tilde{x}, \tilde{y})}{d_{2}^{+}}\left(1-z_{1}\right)\right] \\
& A_{1} \tilde{x}+B_{1} \tilde{y} \leq b_{1} \\
& \max \quad \sum_{i=1}^{2} \xi_{f_{i}} \\
& \text { subject to } \quad \xi_{f_{i}} \leq 1-\left[\frac{f_{i}(\tilde{x}, \tilde{y})-g_{i}^{(1)}}{d_{i 1}^{-}} k_{1}+\frac{f_{i}(\tilde{x}, \tilde{y})-g_{i}^{(2)}}{d_{i 2}^{-}}\left(1-k_{1}\right)\right] \\
& \xi_{f_{i}} \leq 1-\left[\frac{g_{i}^{(1)}-f_{i}(\tilde{x}, \tilde{y})}{d_{i 1}^{+}} k_{1}+\frac{g_{i}^{(2)}-f_{i}(\tilde{x}, \tilde{y})}{d_{i 2}^{+}}\left(1-k_{1}\right)\right] \\
& A_{2} \tilde{x}+B_{2} \tilde{y} \leq b_{2} \\
& \mu_{F_{i}} \geq 0, \xi_{f_{i}} \geq 0, \\
& \tilde{x} \geq 0, \tilde{y} \geq 0 ; i=1,2 ; r=1,2, \ldots, p .
\end{aligned}
$$

Case 2. When there are three fuzzy aspiration goals to choose the FBOBPP will be 


$$
\begin{aligned}
& \max \quad \sum_{i=1}^{2} \mu_{F_{i}} \\
& \text { subject to } \quad \mu_{F_{i}} \leq 1-\left[\frac{F_{i}(\tilde{x}, \tilde{y})-G_{i}^{(1)}}{d_{i 1}^{-}} z_{1} z_{2}+\frac{F_{i}(\tilde{x}, \tilde{y})-G_{i}^{(2)}}{d_{i 2}^{-}}\left(1-z_{1}\right) z_{2}+\right. \\
& \left.+\frac{F_{i}(\tilde{x}, \tilde{y})-G_{i}^{(3)}}{d_{i 3}^{-}} z_{1}\left(1-z_{2}\right)\right] \\
& \mu_{F_{i}} \leq 1-\left[\frac{G_{i}^{(1)}-F_{i}(\tilde{x}, \tilde{y})}{d_{i 1}^{+}} z_{1} z_{2}+\frac{G_{i}^{(2)}-F_{i}(\tilde{x}, \tilde{y})}{d_{i 2}^{+}}\left(1-z_{1}\right) z_{2}+\right. \\
& \left.+\frac{G_{i}^{(3)}-F_{i}(\tilde{x}, \tilde{y})}{d_{i 3}^{+}} z_{1}\left(1-z_{2}\right)\right] \\
& A_{1} \tilde{x}+B_{1} \tilde{y} \leq b_{1} \\
& \max \quad \sum_{i=1}^{2} \xi_{f_{i}} \\
& \text { subject to } \quad \xi_{f_{i}} \leq 1-\left[\frac{f_{i}(\tilde{x}, \tilde{y})-g_{i}^{(1)}}{d_{i 1}^{-}} k_{1} k_{2}+\frac{f_{i}(\tilde{x}, \tilde{y})-g_{i}^{(2)}}{d_{i 2}^{-}}\left(1-k_{1}\right) k_{2}+\right. \\
& \left.+\frac{f_{i}(\tilde{x}, \tilde{y})-g_{i}^{(3)}}{d_{i 3}^{-}} k_{1}\left(1-k_{2}\right)\right] \\
& \xi_{f_{i}} \leq 1-\left[\frac{g_{i}^{(1)}-f_{i}(\tilde{x}, \tilde{y})}{d_{i 1}^{+}} k_{1} k_{2}+\frac{g_{i}^{(2)}-f_{i}(\tilde{x}, \tilde{y})}{d_{i 2}^{+}}\left(1-k_{1}\right) k_{2}+\right. \\
& \left.+\frac{g_{i}^{(3)}-f_{i}(\tilde{x}, \tilde{y})}{d_{i 3}^{+}} k_{1}\left(1-k_{2}\right)\right] \\
& A_{2} \tilde{x}+B_{2} \tilde{y} \leq b_{2} \\
& \mu_{F_{i}} \geq 0, \xi_{f_{i}} \geq 0, \\
& \tilde{x} \geq 0, \tilde{y} \geq 0 ; i=1,2 ; r=1,2, \ldots, p .
\end{aligned}
$$

where $d_{i r}^{+}$and $d_{i r}^{-}, i=1,2 ; r=1,2, \ldots p$ are the positive and negative deviation of $r^{\text {th }}$ choice of fuzzy goal from the optimum value of the $i^{t h}$ objective respectively. We see a binary function is associated with these cases and we may generalize the term as a function of $\Omega_{i}(z, k)$, then the generalized FBOBPP may be written in precise as 


$$
\left.\begin{array}{cc}
\max & \sum_{i=1}^{2} \mu_{F_{i}} \\
\text { subject to } \mu_{F_{i}} \leq 1- & {\left[\sum_{r=1}^{m} \frac{F_{i}(\tilde{x} \tilde{y})-G_{i}^{(r)}}{d_{i r}^{-}} \Omega_{i}(z, k)\right]} \\
\mu_{F_{i}} \leq 1- & {\left[\sum_{r=1}^{m} \frac{G_{i}^{(r)}-F_{i}(\tilde{x}, \tilde{y})}{d_{i r}^{+}} \Omega_{i}(z, k)\right]} \\
& A_{1} \tilde{x}+B_{1} \tilde{y} \leq b_{1}
\end{array}\right]
$$

where the membership function for $i^{\text {th }}$ objective function in first level is given by

$$
\mu_{F_{i}}= \begin{cases}0 & \text { if } F_{i}(\tilde{x}, \tilde{y}) \leq G_{i}^{(r)}+d_{i r}^{-} \\ 1-\left[\frac{F_{i}(\tilde{x}, \tilde{y})-G_{i}^{(r)}}{d_{i r}^{-}} \Omega_{i}(z, k)\right] & \text { if } G_{i}^{(r)} \leq F_{i}(\tilde{x}, \tilde{y}) \leq G_{i}^{(r)}+d_{i r}^{-} \\ 1 & \text { if } F_{i}(\tilde{x}, \tilde{y})=G_{i}^{(r)} ; i=1,2 ; r=1,2, \ldots p \\ 1-\left[\frac{G_{i}^{(r)}-F_{i}(\tilde{x}, \tilde{y})}{d_{i r}^{+}} \Omega_{i}(z, k)\right] & \text { if } G_{i}^{(r)}-d_{i r}^{+} \leq F_{i}(\tilde{x}, \tilde{y}) \leq G_{i}^{(r)} \\ 0 & \text { if otherwise }\end{cases}
$$

and membership function for $i^{t h}$ objective function in second level is

$$
\xi_{f_{i}}= \begin{cases}0 & \text { if } F_{i}(\tilde{x}, \tilde{y}) \leq g_{i}^{(r)}+d_{i r}^{-} \\ 1-\left[\frac{f_{i}(\tilde{x}, \tilde{y})-g_{i}^{(r)}}{d_{i r}^{-}} \Omega_{i}(z, k)\right] & \text { if } g_{i}^{(r)} \leq g_{i}^{(r)}+d_{i r}^{-} \\ 1 & \text { if } F_{i}(\tilde{x}, \tilde{y})=g_{i}^{(r)} ; i=1,2 ; r=1,2, \ldots p \\ 1-\left[\frac{g_{i}^{(r)}-f_{i}(\tilde{x}, \tilde{y})}{d_{i r}^{+}} \Omega_{i}(z, k)\right] & \text { if } g_{i}^{(r)}-d_{i r}^{+} \leq g_{i}^{(r)} \\ 0 & \text { if otherwise. }\end{cases}
$$




\section{SOLUTION OF BOBPP WITH MULTIPLE FUZZY ASPIRATION GOALS}

Now, we adopt the two level Stackelberg function and fuzzy decision model of Sakawa [1993a] to solve the above model of BOBPP with multiple fuzzy aspiration goals. According to this method as given by Emam [2006] the FLDM gets a satisfactory acceptable solution. Then decision variable and some goal are give to FLDM with some room for SLDM to get an optimal solution which is closest to the optimal solution of FLDM.

\subsection{Solution of the FLDM problem}

The FLDM obtains the optimal individual solution, say $F_{i}^{*}$ of all the objectives in upper level and solves the given problem.

Now we can get the solution of the FLDM problem by solving the following problem

$$
\begin{array}{cc}
\text { Max } & \sum_{i=1}^{2} \mu_{F_{i}} \\
\text { subject to } \quad \mu_{F_{i}} \leq 1-\left[\sum_{r=1}^{p} \frac{F_{i}(\tilde{x}, \tilde{y})-G_{i}^{(r)}}{d_{i r}^{-}} \Omega_{i}(z, k)\right] \\
\mu_{F_{i}} \leq 1-\left[\sum_{r=1}^{p} \frac{G_{i}^{(r)}-F_{i}(\tilde{x} \tilde{y})}{d_{i r}^{+}} \Omega_{i}(z, k)\right] \\
A_{1} \tilde{x}+B_{1} \tilde{y} \leq b_{1} \\
A_{2} \tilde{x}+B_{2} \tilde{y} \leq b_{2} \\
0 \geq \mu_{F_{i}} \leq 1 ; \quad i=1,2, \ldots, m \\
\sum_{j=1}^{n} z_{j} \geq 0 ; \quad j=1,2, \ldots, n
\end{array}
$$

whose solution is assumed to be

$\tilde{x}^{F}, \tilde{y}^{F}, F_{i}^{F} ; i=1,2, \ldots, m$.

\subsection{Solution of the SLDM problem}

In similar way the SLDM solves his/her problem and denote its individual optimal solution as $f_{i}^{*}$. Again the solution of the SLDM's problem is obtained by solving the problem given below 


$$
\begin{array}{cc}
\text { Max } & \sum_{i=1}^{2} \xi_{f_{i}} \\
\text { subject to } \quad \xi_{f_{i}} \leq 1-\left[\sum_{r=1}^{p} \frac{f_{i}(\tilde{x}, \tilde{y})-g_{i}^{(r)}}{d_{i r}^{-}} \Omega_{i}(z, k)\right] \\
\xi_{f_{i}} \leq 1-\left[\sum_{r=1}^{p} \frac{g_{i}^{(r)}-f_{i}(\tilde{x}, \tilde{y})}{d_{i r}^{+}} \Omega_{i}(z, k)\right] \\
A_{1} \tilde{x}+B_{1} \tilde{y} \leq b_{1} \\
A_{2} \tilde{x}+B_{2} \tilde{y} \leq b_{2} \\
0 \geq \xi_{f_{i}} \leq 1 ; \quad i=1,2, \ldots, m \\
\sum_{j=1}^{n} k_{j} \geq 0 ; \quad j=1,2, \ldots, n
\end{array}
$$

whose solution is assumed to be $\tilde{x}^{S}, \tilde{y}^{S}, F_{i}^{S} ; i=1,2, \ldots, m$

Now the solution of the FLDM and SLDM are disclosed. However, two solutions are usually different because of nature between both levels objective functions. The FLDM knows that using the optimal decisions $\tilde{x}^{F}$ as a control factor for the SLDM is not practical. It is more reasonable to have some tolerance that gives the SLDM an extent feasible region to search for his/her optimal solution, and also reduce searching time or interactions.

In this way, the range of decision variables $\tilde{x}$ should be around $\tilde{x}^{F}$ with maximum tolerances $t$ and the following membership function specify $\tilde{x}^{F}$ as

$$
\mu_{\tilde{x}}=\frac{\tilde{x}-\left(\tilde{x}^{F}-t\right)}{t} \quad \tilde{x}^{F}-t \leq \tilde{x} \leq \tilde{x}^{F}
$$

where $\tilde{x}^{F}$ is the most preferred solution.

First, the FLDM goals may reasonably consider all $F_{i} \geq F_{i}^{F}, i=1,2, \ldots, m$ absolutely acceptable and all $F_{i}(\tilde{x}, \tilde{y})<F_{i}^{\prime}=F_{i}\left(\tilde{x}^{S}, \tilde{y}^{S}\right), i=1,2, \ldots, m$ absolutely unacceptable, and that the preference with $\left[F_{i}^{\prime}, F_{i}^{F}, i=1,2, \ldots, m\right]$ is linearly increasing. This is due to the fact that the SLDM obtained the optimum at $\left(\tilde{x}^{S}, \tilde{y}^{S}\right)$, which in turn provides the FLDM the objective function values $F_{i}^{\prime}$, makes any $F_{i}(\tilde{x}, \tilde{y})<F_{i}^{\prime}, i=1,2, \ldots, m$ unattractive in practice.

The following membership functions of the FLDM can be stated as

$$
\mu_{i}^{\prime}= \begin{cases}0 & \text { if } F_{i}(\tilde{x}, \tilde{y})>F_{i}^{F} \\ 1-\left[\frac{F_{i}(\tilde{x}, \tilde{y})-F_{i}^{\prime}}{F_{i}^{F}-F_{i}^{\prime}}\right] & \text { if } F_{i}^{\prime} \leq F_{i}(\tilde{x}, \tilde{y}) \leq F_{i}^{F} \\ 1 & \text { if } F_{i}(\tilde{x}, \tilde{y})<F_{i}^{\prime} \quad i=1,2, \ldots, m\end{cases}
$$


Second, the SLDM may be willing to build a membership function for his/her objective functions, so that he/she can rate the satisfaction of each potential solution. In this way, the SLDM has the following membership functions for his/her goals:

$$
\xi_{i}^{\prime}= \begin{cases}0 & \text { if } f_{i}(\tilde{x}, \tilde{y})>f_{i}^{F} \\ 1-\left[\frac{f_{i}(\tilde{x}, \tilde{y})-f_{i}^{\prime}}{f_{i}^{f}-S_{i}^{\prime}}\right] & \text { if } f_{i}^{\prime} \leq f_{i}(\tilde{x}, \tilde{y}) \leq f_{i}^{S} \\ 1 & \text { if } f_{i}(\tilde{x}, \tilde{y})<f_{i}^{\prime} \quad i=1,2, \ldots, m\end{cases}
$$

where $f_{i}^{\prime}=f_{i}\left(\tilde{x}^{F}, \tilde{y}^{F}\right)$

Finally, in order to generate the satisfactory solution, which is also a Pareto optimal solution with overall satisfaction for all DMs, solve the following Tchebycheff problem.

$$
\begin{array}{cc}
\max _{\tilde{x} \in X} & \delta \\
\text { subject to } & \frac{\tilde{x}-\left(\tilde{x}^{F}+t_{1}\right)}{t} \geq \delta \\
A_{1} \tilde{x}+B_{1} \tilde{y} \leq b_{1} \\
A_{2} \tilde{x}+B_{2} \tilde{y} \leq b_{2} \\
\xi_{i}^{\prime} \leq 1-\left[\frac{F_{i}(\tilde{x}, \tilde{y})-F_{i}^{\prime}}{F_{i}^{F}-F_{i}^{\prime}}\right] \\
\mu_{i}^{\prime} \leq 1-\left[\frac{f_{i}(\tilde{x}, \tilde{y})-f_{i}^{\prime}}{f_{i}^{f}-S_{i}^{\prime}}\right] \\
\sum_{j=1}^{n} z_{j} \geq 0 \\
\sum_{j=1}^{n} k_{j} \geq 0 \\
0 \geq \delta \geq 1 \\
0 \geq \mu_{i} \leq 1 \\
0 \geq \xi_{i} \leq 1 \\
t>0 ; \quad i=1,2, \ldots, m ; \quad j=1,2, \ldots, n
\end{array}
$$

where $\delta$ is the overall satisfaction.

After solving the above equation if the FLDM is satisfied with solution then satisfactory solution is reached. Otherwise, the FLDM should provide new membership functions for the control variable and objectives to the SLDM until a satisfactory solution is reached. 


\section{NUMERICAL EXAMPLE}

Consider a BOBPP with multiple fuzzy aspiration goals given below

$$
\left.\begin{array}{ccc}
\max _{x \in X} F_{1} & =2 \tilde{20} \tilde{x}+\tilde{30} \tilde{y} & \approx 240,275 \\
F_{2} & =\tilde{30} \tilde{x}+\tilde{20} \tilde{y} & \approx 210,260,290
\end{array}\right\} \text { where } \tilde{y} \text { solves }
$$

$$
\begin{aligned}
& \text { subject to } \quad 11 \tilde{x}+12 \tilde{y} \leq 130 \\
& \max _{y \in Y} f_{1} \quad=\tilde{9} \tilde{x}+\tilde{2} 3 \tilde{y} \quad \approx 160,180,200 \\
& f_{2}=\tilde{10} \tilde{x}+\tilde{24} \tilde{y} \quad \approx 160,185 \\
& \text { subject to } 16 \tilde{x}+17 \tilde{y} \leq 120 \\
& -12 \tilde{x}+9 \tilde{y} \leq 35 \\
& \tilde{x} \geq 0, \tilde{y} \geq 0
\end{aligned}
$$

Let the fuzzy cost coefficients and decision variables are symmetric trapezoidal fuzzy numbers where

$\tilde{20}=16,20,22,22 ; \tilde{30}=15,30,45,45 ; \tilde{9}=7,9,12,12$

$\tilde{23}=20,23,25,25 ; \tilde{10}=7,10,12,12 ; \tilde{24}=20,24,28,28$

Thus, the above problem in terms of symmetric trapezoidal fuzzy numbers can be rewritten as

$$
\begin{aligned}
\max _{x \in X} F_{1}= & (16,20,22,22) \otimes\left(x_{1}, x_{2}, x_{3}, x_{3}\right)+ \\
& +(15,30,45,45) \otimes\left(y_{1}, y_{2}, y_{3}, y_{3}\right) \quad \approx 240,275 \\
\max _{x \in X} F_{2}= & (15,30,45,45) \otimes\left(x_{1}, x_{2}, x_{3}, x_{3}\right)+ \\
& +(16,20,22,22) \otimes\left(y_{1}, y_{2}, y_{3}, y_{3}\right) \quad \approx 210,260,290
\end{aligned}
$$




$$
\begin{aligned}
& \text { subject to } 11 \otimes\left(x_{1}, x_{2}, x_{3}, x_{3}\right)+12 \otimes\left(y_{1}, y_{2}, y_{3}, y_{3}\right) \leq 130 \\
& \begin{aligned}
\max _{y \in Y} f_{1} & =(7,9,12,12) \otimes\left(x_{1}, x_{2}, x_{3}, x_{3}\right)+ \\
& +(20,23,25,25) \otimes\left(y_{1}, y_{2}, y_{3}, y_{3}\right) \quad \approx 160,180,200
\end{aligned} \\
& \max _{y \in Y} f_{2} \quad=(7,10,12,12) \otimes\left(x_{1}, x_{2}, x_{3}, x_{3}\right)+ \\
& +(20,24,28,28) \otimes\left(y_{1}, y_{2}, y_{3}, y_{3}\right) \quad \approx 160,185
\end{aligned}
$$

subject to $16 \otimes\left(x_{1}, x_{2}, x_{3}, x_{3}\right)+17 \otimes\left(y_{1}, y_{2}, y_{3}, y_{3}\right) \leq 120$

$$
\begin{gathered}
-12 \otimes\left(x_{1}, x_{2}, x_{3}, x_{3}\right)+9 \otimes\left(y_{1}, y_{2}, y_{3}, y_{3}\right) \leq 35 \\
\left(x_{1}, x_{2}, x_{3}, x_{3}\right) \geq 0,\left(y_{1}, y_{2}, y_{3}, y_{3}\right) \geq 0
\end{gathered}
$$

The symmetric trapezoidal individual optimal values of the objectives of FLDM, SLDM, $\tilde{x}$ and $\tilde{y}$ are given in table below

\begin{tabular}{c|c|c|c|c}
$F_{1}^{*}$ & 202.5463 & 301.8519 & 201.0311 & 201.0311 \\
\hline$F_{2}^{*}$ & 198.7609 & 274.6685 & 176.2234 & 176.2234 \\
\hline$f_{1}^{*}$ & 209.2438 & 216.5021 & 132.7426 & 132.7426 \\
\hline$f_{2}^{*}$ & 192.0062 & 222.9630 & 134.9465 & 134.9465 \\
\hline
\end{tabular}

Table 1: Individual optimal values of each objective function

Now, using eq. (9) the FLDM's problem is as given below

$\max$

$$
\mu_{1}+\mu_{2}
$$

subject to

$$
\begin{gathered}
\mu_{1} \leq 1-\left[\frac{F_{1}(\tilde{x}, \tilde{y})-240}{F_{1}^{*}-240} z_{1}+\frac{F_{1}(\tilde{x}, \tilde{y})-275}{F_{1}^{*}-275}\left(1-z_{1}\right)\right] \\
\mu_{1} \leq 1-\left[\frac{240-F_{1}(\tilde{x}, \tilde{y})}{F_{1}^{*}-240} z_{1}+\frac{275-F_{1}(\tilde{x}, \tilde{y})}{F_{1}^{*}-275}\left(1-z_{1}\right)\right] \\
\mu_{2} \leq 1-\left[\frac{F_{2}(\tilde{x}, \tilde{y})-210}{F_{2}^{*}-210} z_{2} z_{3}+\frac{F_{2}(\tilde{x}, \tilde{y})-260}{F_{2}^{*}-260} z_{2}\left(1-z_{3}\right) \frac{F_{2}(\tilde{x}, \tilde{y})-290}{F_{2}^{*}-290}\left(1-z_{2}\right) z_{3}\right] \\
\mu_{2} \leq 1-\left[\frac{210-F_{2}(\tilde{x}, \tilde{y})}{F_{2}^{*}-210} z_{2} z_{3}+\frac{260-F_{2}(\tilde{x}, \tilde{y})}{F_{2}^{*}-260} z_{2}\left(1-z_{3}\right) \frac{290-F_{2}(\tilde{x}, \tilde{y})}{F_{2}^{*}-290}\left(1-z_{2}\right) z_{3}\right] \\
11 \tilde{x}+12 \tilde{y} \leq 130 \\
16 \tilde{x}+7 \tilde{y} \leq 120 \\
-12 \tilde{x}+9 \tilde{y} \leq 35 \\
z_{2}+z_{3} \geq 1 \\
1 \geq \mu_{1}, \mu_{2} \geq 0 .
\end{gathered}
$$




\begin{tabular}{l|l|l|l|l}
$F_{1}^{F}$ & 202.5463 & 204.8644 & 228.7890 & 228.7890 \\
\hline$F_{2}^{F}$ & 193.9403 & 180.7770 & 274.5718 & 274.5718 \\
\hline$f_{1}^{F}$ & 202.5463 & 148.5584 & 125.3088 & 125.3088 \\
\hline$f_{2}^{F}$ & 192.0062 & 150.9034 & 134.9834 & 134.9834 \\
\hline
\end{tabular}

Table 2: Values for FLDM's solution

Again, using eq. (10) the solution of the SLDM's problem can be obtained from

$\operatorname{Max}$

$$
\begin{gathered}
\xi_{1}+\xi_{2} \\
\xi_{1} \leq 1-\left[\frac{f_{1}(\tilde{x}, \tilde{y})-160}{f_{1}^{*}-160} k_{1} k_{2}+\frac{f_{1}(\tilde{x}, \tilde{y})-180}{f_{1}^{*}-180} k_{1}\left(1-k_{2}\right) \frac{f_{1}(\tilde{x}, \tilde{y})-200}{f_{1}^{*}-200}\left(1-k_{1}\right) k_{2}\right] \\
\xi_{1} \leq 1-\left[\frac{160-f_{1}(\tilde{x}, \tilde{y})}{f_{1}^{*}-160} k_{1} k_{2}+\frac{180-f_{1}(\tilde{x}, \tilde{y})}{f_{1}^{*}-180} k_{1}\left(1-k_{2}\right) \frac{200-f_{1}(\tilde{x}, \tilde{y})}{f_{1}^{*}-200}\left(1-k_{1}\right) k_{2}\right] \\
\xi_{2} \leq 1-\left[\frac{f_{2}(\tilde{x}, \tilde{y})-160}{f_{2}^{*}-160} k_{3}+\frac{f_{2}(\tilde{x}, \tilde{y})-185}{f_{2}^{*}-185}\left(1-k_{3}\right)\right] \\
\xi_{2} \leq 1-\left[\frac{160-f_{2}(\tilde{x}, \tilde{y})}{f_{2}^{*}-160} k_{3}+\frac{185-f_{2}(\tilde{x}, \tilde{y})}{f_{2}^{*}-185}\left(1-k_{3}\right)\right] \\
11 \tilde{x}+12 \tilde{y} \leq 130 \\
16 \tilde{x}+7 \tilde{y} \leq 120 \\
-12 \tilde{x}+9 \tilde{y} \leq 35 \\
k_{1}+k_{2} \geq 1 \\
1 \geq \xi_{1}, \xi_{2} \geq 0 .
\end{gathered}
$$

subject to

\begin{tabular}{c|c|c|c|c}
$F_{1}^{S}$ & 179.7286 & 167.7180 & 319.4375 & 319.4375 \\
\hline$F_{2}^{S}$ & 168.6757 & 147.5871 & 263.8660 & 263.8660 \\
\hline$f_{1}^{S}$ & 188.7443 & 120.1569 & 196.2188 & 196.2188 \\
\hline$f_{2}^{S}$ & 172.6852 & 122.7885 & 211.2017 & 211.2017 \\
\hline
\end{tabular}

Table 3: Values for SLDM's solution

Finally, using eq. (11), eq.(12) and eq.(13) and assuming the FLDM's control decision $x_{1}^{F}$ around 0 with tolerance 1 , the pareto-optimal solution which is satisfactory to both DM's is obtained solving the Fuzzy programming problem in eq.(14).

Now the trapezoidal symmetrical fuzzy optimal values are converted into their 


\begin{tabular}{c|c|c|c|c}
$F_{1}^{\prime}$ & 179.7286 & 176.785 & 218.785 & 218.785 \\
\hline$F_{2}^{\prime}$ & 168.6757 & 145.995 & 233.0375 & 233.0375 \\
\hline$f_{1}^{\prime}$ & 188.7443 & 141.3384 & 130.5005 & 130.5005 \\
\hline$f_{2}^{\prime}$ & 172.6852 & 144.6666 & 138.4983 & 138.4983
\end{tabular}

Table 4: Trapezoidal Fuzzy values for BOBLPP

respective crisp values

i.e. $F_{1}^{\prime}=178.2568, F_{2}^{\prime}=157.33535, F_{3}^{\prime}=165.04135$ and $F_{4}^{\prime}=158.6756$

Now when these crisp goals have been obtained the BOBLPP becomes

$$
\left.\begin{array}{ccc}
\max _{x \in X} F_{1} & =20 x+30 y & =178.2568 \\
F_{2} & =30 x+20 y & =157.33535
\end{array}\right\} \text { where } \tilde{y} \text { solves }
$$

subject to $11 x+12 y \leq 130$

$$
\begin{aligned}
\max _{y \in Y} f_{1} & =9 x+23 y & =165.04135 \\
f_{2} & =10 x+24 y & =158.6756
\end{aligned}
$$

subject to $16 x+17 y \leq 120$

$$
\begin{gathered}
-12 x+9 y \leq 35 \\
x \geq 0, y \geq 0
\end{gathered}
$$

So the compromise solution finally obtained is $\left(x^{C}, y^{C}\right)=(1.403957,5.760832)$

$$
\begin{aligned}
& \left(F_{1}^{C}, F_{2}^{C}\right)=(200.9041,157.3354) \\
& \left(f_{1}^{C}, f_{2}^{C}\right)=(145.1347,152.2995)
\end{aligned}
$$

Figure (1) illustrates pareto optimal solution of the above problem.

If the FLDM is satisinñed with the above solution, then a satisfactory solution is reached. Otherwise, one should provide new membership functions for the control variable and objectives to the SLDM, who also should provide new membership function for the control variable and objectives to the SLDM until a satisfactory solution is reached. 


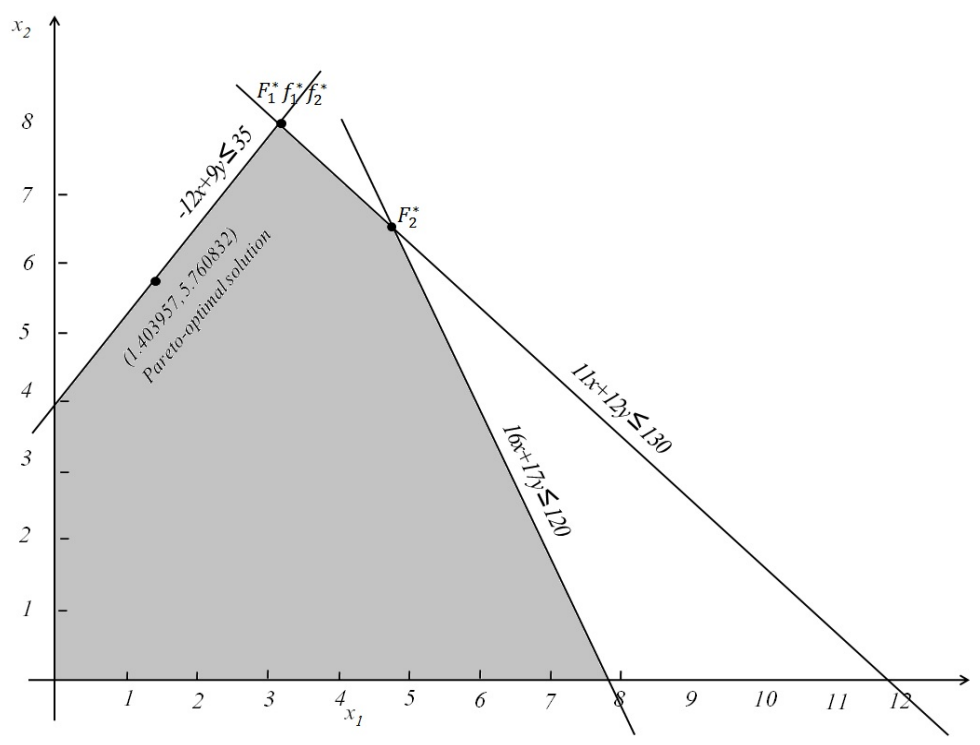

Figure 1: A Pareto Optimal Solution

\section{CONCLUSION}

In this paper the author considered a practical situation of multiple fuzzy choices of goals for decision makers, fuzzy cost coefficients and fuzzy decision variables with their own set of objectives. A two level problem with two objectives at each level and fuzzy aspiration goals is formulated as a fuzzy bi-objective bilevel programming problem (FBOBPP). The fuzzy cost coefficients and fuzzy decision variables are considered symmetric trapezoidal fuzzy numbers. Using ranking function the model is converted into a crisp model. The FBOBPP is then solved using two planner stackelberg function and fuzzy decision model with tolerance function. A comprehensive study of bi-objective bilevel programming problem in case of fuzzy parameters has been done in this paper. Finally, a pareto-optimal solution for decision maker's of bilevel problem is obtained. The problem can be further extended as a multi-objective multilevel programming problem with multiple fuzzy aspiration goals. 


\section{REFERENCES}

T. Allahviranloo, F. H. Lofti, M. K. Kiasary, N.A. Kiani, and L. Alizadeh. Solving fully fuzzy linear programming problem by the ranking function. Applied Mathematical Sciences, 2(1):19-32, 2008.

G. Anandalingam. A mathematical programming model of decentralized multi-level systems. Journal of Operational Research Society, 39:1021âĂŞ1033, 1988.

J.F. Bard. A grid search algorithm for the linear bilevel programming problem. 14-th Annual Meeting of American Institude for Decision Science, San Francisco, CA.2:256-258, 1982.

W. Bialas, M.H. Karwan, and J Shaw. A parametric complementary pivot approach for two-level linear programming. Technical Report, State University of New York at Buffalo, Operations Research, 80-2, 1980.

M. P. Biswal and S. Acharya. Transformation of a multi-choice linear programming problem. Applied Mathematics and Computation, 210:182-188, 2009.

M. P. Biswal and S. Acharya. Solving multi-choice linear programming problems by interpolating polynomials. Mathematical and Computer Modelling, 54:1405-1412, 2011.

C. T. Chang. Multi-choice goal programming. Omega, 35(4):389-396, 2007.

O.E. Emam. A fuzzy approach for bi-level integer non-linear programming problem. Applied Mathematics and Computation, 172:62âĂŞ71, 2006.

R. Ezzati, E. Khorram, and R. Enayati. A algorithm to solve fully fuzzy linear programming problems using the molp problem. Applied Mathematical Modelling, In Press, 2013.

K. Ganesan and P. Veeramani. Fuzzy linear programs with trapezoidal fuzzy numbers. Annals of Operations Research, 143:305-315, 2006.

W. C. Healy. Multiple choice programming. operations research. International journal of Mathematics and Applied Statistics, 12:122-138, 1964.

F. Hiller and G. Lieberman. Introduction to operations research. McGraw-Hill, New York, 1990.

Masahiro Inuiguchi and Jaroslav Ramık. Possibilistic linear programming: a brief review of fuzzy mathematical programming and a comparison with stochastic programming in portfolio selection problem. Fuzzy sets and systems, 111(1):3-28, 2000.

B. Liu. Stackelberg-nash equilibrium for multi-level programming with multi-follows using genetic algorithms. Computers math applications, 37,7:79-89, 1998.

J. Lu, C. Shi, and G. Zhang. An extended kth-best approach for linear bilevel programming. Applied Mathematics and Computation, 164:843-855, 2005.

MK Luhandjula. Multiple objective programming problems with possibilistic coefficients. Fuzzy Sets and Systems, 21(2):135-145, 1987.

R. Mathieu, L. Pittard, and G. Anandalingam. Genetic algorithm based approach to bilevel linear ptogramming. R. A. I. R. O Researche operationelle, 28:1-21, 1994.

Heinrich Rommelfanger. Fuzzy linear programming and applications. European journal of operational research, 92(3):512-527, 1996.

Heinrich Rommelfanger. The advantages of fuzzy optimization models in practical use. Fuzzy Optimization and Decision Making, 3(4):295-309, 2004.

M Sakawa. Interactive fuzzy goal programming for multiobjective nonlinear problems and its application to water quality management. Control and Cybernetics, 13(2):217-228, 1984.

M. Sakawa. Fuzzy sets and interactive multi-objective optimization. Plenum Press, New York, 1993 a.

Masatoshi Sakawa. Fuzzy sets and interactive multiobjective optimization. Plenum New York, 1993b.

Fumiko Seo and Masatoshi Sakawa. Multiple criteria decision analysis in regional planning: concepts, methods and applications. Reidel Dordrecht, The Netherlands, 1988.

C. Shi, J. Lu, and G. Zhang. An extended kuhn-tucker approach for linear bilevel programming. Applied Mathematics and Computation, 162:51-63, 2005.

Roman Slowinski and Jacques Teghem. Stochastic vs. fuzzy approaches to multiobjective mathematical programming under uncertainty. Kluwer Academic Publishers, 1990. 
Behzad Bankian Tabrizi, Kamran Shahanaghi, and M. Saeed Jabalameli. Fuzzy multi-choice goal programming. Applied Mathematical Modelling, 36:1415-1420, 2012.

Jea Teghem Jr, D Dufrane, M Thauvoye, and P Kunsch. Strange: an interactive method for multi-objective linear programming under uncertainty. European Journal of Operational Research, 26(1):65-82, 1986.

W.F. Bialas U.P. Wen. The hybrid algorithm for solving the three-level linear programming problem. Computers and Operations Research, 13:367âĂŞ377, 1986.

H-J Zimmermann. Fuzzy programming and linear programming with several objective functions. Fuzzy sets and systems, 1(1):45-55, 1978.

\section{Sanam Haseen}

Department of Statistics and Operations Research

Aligarh Muslim University,

Aligarh, India

sanam.haseen@gmail.com

\section{Abdul Bari}

Department of Statistics and Operations Research

Aligarh Muslim University,

Aligarh, India 